\title{
Importancia y caracterización de frutales criollos en dos comunidades de Tuxpan, Veracruz
}

\section{Creole fruits importance and characterization in two communities of Tuxpan, Veracruz}

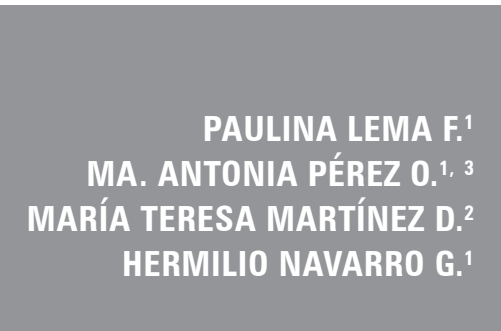

Frutos verdes y maduros de caimito.

Foto: P. Lema Franco

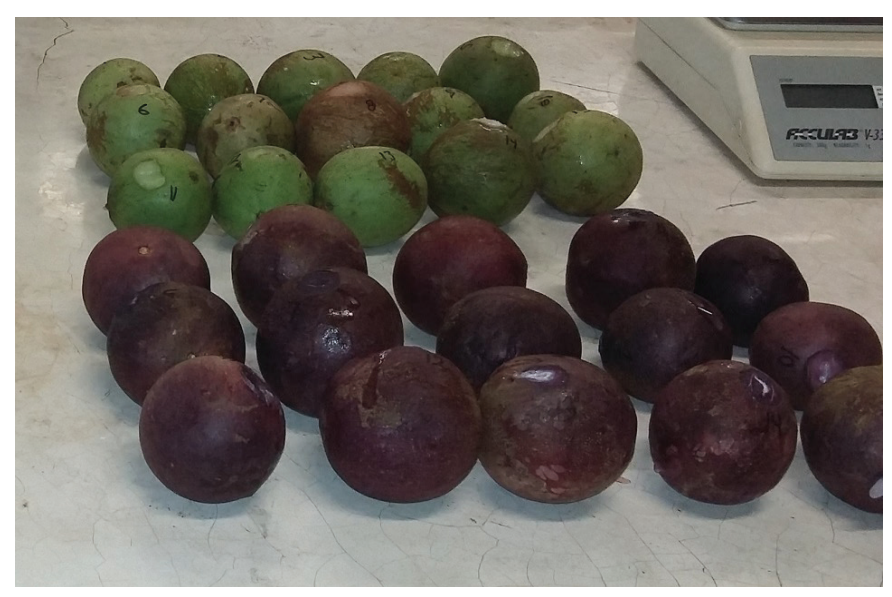

\section{RESUMEN}

Los solares (huertos) en las comunidades de Congregación (C) y Ejido (E) Juana Moza de Tuxpan, Veracruz (México) se caracterizan por tener diversidad de especies vegetales. Los árboles frutales son un componente importante de esa diversidad, ya que tienen usos alimenticios y son fuentes de ingresos, entre otros. Sin embargo, las características físicas y químicas de los frutales criollos han sido poco estudiadas. La presente investigación se llevó a cabo con la finalidad de evaluar la importancia y contribuir a la caracterización físico-química de cinco especies de frutales criollos: caimito verde y morado (Chrysophyllum cainito L.), chalahuite (Inga paterno Harms), guaya (Melicoccus bijugatus Jacq.), zapote domingo (Mammea americana L.) y jobo (Spondias mombin L.). Se seleccionó una muestra de 63 productores (24 en E y 39 en C), a quienes se les aplicó una encuesta. Adicionalmente se muestrearon 30 solares (20 en C y 10 en E), que estuvieran en época de cosecha, donde se recolectaron 10 frutos de diferentes partes del árbol para evaluar el peso, diámetro ecuatorial y longitud del fruto; se tomaron 3 frutos por solar y especie para determinar color, firmeza, grados Brix y acidez, en el laboratorio. Los resultados indican que caimito, chalahuite y guaya se aprovechan como fruta fresca; el zapote domingo y el jobo se usan para preparar bebidas refrescantes y licores. Adicionalmente con el jobo se elaboran "curados" (bebidas alcohólicas locales). Las semillas del chalahuite se consumen en cocción. La caracterización dio como resultado que variables como peso, longitud y diámetro son similares en las dos comunidades, lo que muestra el potencial regional; sin embargo, el contenido de grados Brix y acidez presentaron diferencias estadísticas significativas $(P \leq 0,05)$, aspectos importantes para el destino de la misma, ya sea como fruta fresca o para la elaboración de otros productos.

Postgrado en Estudios del Desarrollo Rural, Colegio de Postgraduados, Montecillo (México). ORCID Lema F., P.: 00000003-2330-080X; Pérez O., M.A.: 0000-0002-6408-8641; Navarrro G., H.: 0000-0002-8599-3448

2 Departamento de Fitotecnia, Universidad Autónoma Chapingo, Chapingo (México). ORCID Martínez D., M.T.: 00000002-7204-2133

3 Autor para correspondencia. molvera@colpos.mx 
Palabras clave adicionales: conocimiento local, diversidad, especies frutales, poscosecha.

\section{ABSTRACT}

Home-gardens or "Solares" in the communities Congregación and Ejido Juana Moza of Tuxpan, Veracruz are characterized by their high plant diversity. Fruit trees are an important component of this diversity. These fruits are used for food and income, among others. However, their physical and organoleptic characteristics are scarcely documented. This research aimed to characterize and evaluate the economic and nutritional importance of five species of creole fruits: Green and purple caimito (Chrysophyllum cainito L.), chalahuite (Inga paterno Harms), guaya (Melicoccus bijugatus Jacq.), zapote domingo (Mammea americana L.) and jobo (Spondias mombin L.). A sample of 63 heads of household was selected (24 in Ejido and 39 in Congregation Juana Moza), to whom a survey was applied. In addition, 30 lots (20 in C and $10 \mathrm{E}$ ) were sampled at harvest time, where 10 fruits from different parts of the trees were collected to evaluate the fruit diameter and length, and 3 fruits were taken per plot and species to determine the color, firmness, degrees Brix and acidity in a laboratory. The results indicated that caimito, Chalahuite and guaya are used as fresh fruit; zapote domingo and jobo are used to prepare refreshing drinks and liqueurs. In addition, jobo is used to prepare "curados" (a local alcoholic drink) and Chalahuite seeds are cooked and used as food. The characterization of the fruits indicated that variables such as weight, length and diameter were similar in both communities, while the degrees Brix and acidity had statistically significant differences. Local management and use of the fruits are important because families obtain fruit for self-consumption and income from April-September; in addition, the trees are used for shade, fencing, fire wood.

Additional key words: local knowledge; diversity; fruit species; post-harvest.

Fecha de recepción: 02-04-2017 Aprobado para publicación: 30-09-2017

INTRODUCCIÓN

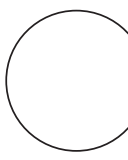

INTRODUCCION

Se tiene conocimiento que en diferentes zonas de México se encuentran alrededor de 1.500 variedades de plantas cultivadas de forma rústica y con fines alimenticios, constituyendo alrededor de una cuarta parte en el consumo al año de los productores rurales (Lascurain et al., 2010). Borys y Leszczyñska (2001) mencionan que existen en México 63 especies de frutales comerciales y 1.220 especies con potencial documentado. Veracruz, se considera uno de los estados con mayor riqueza biológica y diversidad florística (Ambrosio y Avendaño, 1999), ya que se valúan alrededor de 140 variedades de frutas rústicas para el consumo familiar y que también son conocidas como autóctonas, criollas, nativas o cimarronas, entre otras (Lascurain et al., 2010).

Rodríguez et al. (2012) señalan que los frutales criollos son cultivos de tradición y arraigo en muchas zonas productoras de México, que generan sustento para numerosas familias, de las cuales depende su modo de vida. El National Research Council (1989) menciona que es posible encontrar una variedad de frutos nativos que son de particular importancia para campesinos y pequeños agricultores, debido a que pueden ser cultivados fácilmente alrededor de sus casas huertas, o solares y constituyen un recurso importante como alimento y como una pequeña fuente de ingresos por la venta de los mismos. Por lo tanto, es necesario resaltar su importancia y generar conocimiento para su utilización y posible incorporación al mercado mediante pequeñas o medianas agroindustrias con especies frutales localmente conocidas que representan un potencial significativo para la gastronomía (Vernooy, 2003) y que permiten vislumbrar perspectivas $\mathrm{y}$ nichos de mercado a nivel nacional e internacional (Rodríguez et al., 2012).

La venta local de frutos criollos o silvestres representa un ingreso para las familias campesinas de Veracruz, quienes esperan la época de recolección o cosecha de 
frutales para obtener un ingreso extra, y sufragar diversos gastos o bien para tener fruta para autoconsumo. Los frutales criollos representan una opción para disminuir la pobreza en zonas rurales, como parte de la dieta alimenticia mediante el autoconsumo, esto reportado desde tiempos prehispánicos (Lascurain et al., 2010).

Estudios recientes sobre especies frutales criollos resaltan sus propiedades nutritivas (Lascurain et al., 2010; Peña y Ruiz, 2003), sobre la caracterización físico-química de otros (Álvarez et al., 2006; Hernández et al., 2009). Dichos estudios constituyen la base para diseñar y aplicar estrategias para el uso, aprovechamiento y conservación de éstas especies (Martínez 2010; López et al., 2012). Sin embargo, siguen siendo escasos, sobre todo para las especies localizadas al norte del estado de Veracruz. El objetivo de esta investigación fue evaluar la importancia de las cinco especies de frutales criollos encontradas con más frecuencia en los solares de las familias y contribuir a la caracterización físico-química de dichos frutos.

\section{MATERIALES Y MÉTODOS}

El trabajo de campo se realizó durante 2015 (marzo-agosto), en Congregación y Ejido Juana Moza, comunidades pertenecientes al municipio de Tuxpan, Veracruz (205' N; 97²4' O). Se localiza al norte del estado de Veracruz, su cabecera municipal se encuentra ubicada a orillas del río Tuxpan, a $11 \mathrm{~km}$ de su desembocadura en el Golfo de México. Su clima es tropical con una temperatura media anual de $24,9^{\circ} \mathrm{C}$; con lluvias abundantes en el verano y a principios del otoño, con menor intensidad de noviembre a mayo, su precipitación media anual es de 1,241 milímetros. La vegetación es bosque alto tropical, perinifolio (Instituto Nacional de Estadística Geografía e Informática INEGI, 2010).
Las comunidades fueron seleccionadas por presentar la mayor diversidad de especies (frutales, hortalizas, flores, plantas medicinales y animales). A nivel de comunidad se realizó un recorrido por todos los solares para identificar las especies vegetales que en ellos se encontraban. Los frutales encontrados con mayor frecuencia fueron: caimito verde y morado (Chrysophyllum cainito L.), chalahuite (Inga paterno Harms), guaya (Melicoccus bijugatus Jacq.), zapote domingo (Mammea americana L.) y jobo (Spondias mombin L.).

El inventario, las fechas de fructificación, el uso, la importancia y la edad de los frutales se obtuvo mediante una encuesta aplicada a 63 productores $(24$ en Ejido Juana Moza y 39 en Congregación Juana Moza). Con los datos de fructificación reportados, se definieron las fechas para muestreo y análisis de frutos (Tab. 1). La selección de los solares a muestrear fue que tuvieran árboles frutales en fructificación (7-8 años de edad) y que la fruta estuviera lista para su recolección de acuerdo con los criterios del productor.

\section{Recolección y determinación de variables físicas y químicas de los frutos}

En los solares seleccionados (Fig. 1), se tomaron 10 frutos al azar, de diferentes alturas y orientación de la planta para medir las variables de peso, diámetro ecuatorial y longitud; para las variables: color, firmeza, acidez titulable y solidos solubles totales, se tomaron tres frutos por solar (entre 15-24 frutos por especie), los cuales fueron transportados en cajas al laboratorio de Fisiología de Postcosecha de la Universidad Autónoma Chapingo para su acondicionamiento y análisis, el cual se realizó entre 2 a 3 d posteriores a su recolección; manteniendo las muestras en condiciones de refrigeración.

Tabla 1. Fechas de fructificación y recolección de frutales criollas en dos comunidades de Tuxpan, México.

\begin{tabular}{|l|c|c|c|c|c|c|}
\hline \multirow{2}{*}{ Frutal } & \multirow{2}{*}{ Época de fructificación } & \multirow{2}{*}{$\begin{array}{c}\text { Fecha de } \\
\text { recolección }\end{array}$} & \multicolumn{2}{c|}{ Congregación Juana Moza } & \multicolumn{2}{c|}{ Ejido Juana Moza } \\
\cline { 4 - 7 } & & Solares ${ }^{1}$ & Total & \multicolumn{2}{|c|}{ Solares* } & \multicolumn{1}{|c|}{ Total } \\
\hline Caimito & Abril-Mayo & 18-19 Abril & $2,8,11,13,15,17,20$ & 7 & $1,2,4,5,6,7,9,10$ & 8 \\
\hline Guaya & Mayo-Junio & 13-14 Junio & $3,4,5,8,12,14,18,1$ & 8 & $1,2,3,6,9$ & 5 \\
\hline Zapote domingo & Junio-Septiembre & 13-14 Junio & $3,4,10,12,13,14,16,18,19$ & 9 & $1,2,5,6,9,10$ & 6 \\
\hline Chalahuite & Julio-Agosto & 11-12 Julio & $4,5,7,9,17,20$ & 6 & $1,4,5,7,8$ & 5 \\
\hline Jobo & Agosto-Septiembre & 15-16 Agosto & $1,2,6,11,13,16$ & 6 & $1,3,4,8,10$ & 5 \\
\hline
\end{tabular}

${ }^{1}$ Identificador del solar en el muestreo. 


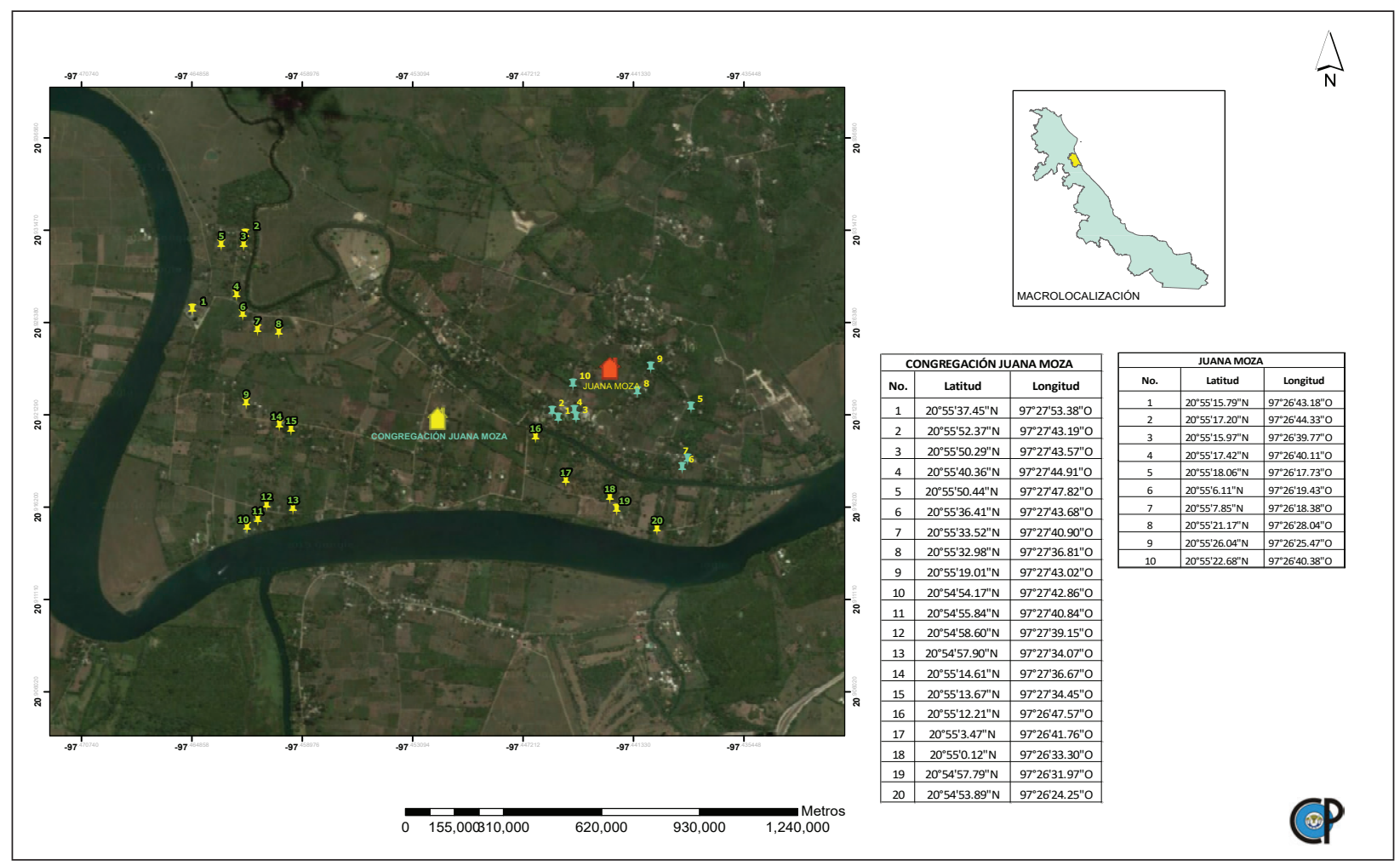

Figura 1. Ubicación de los sitios (solares) de recolección de especies frutales criollas en Congregación y Ejidos Juana Moza, Tuxpan, México.

Los frutos se cosecharon directamente de los árboles utilizando: escalera, tijera y gancho de acuerdo con la altura de los frutales, la forma de cortarla y la susceptibilidad de la fruta. Para la selección de los frutos de zapote domingo, se consideró el criterio de las familias, de tomar los frutos recientemente caídos del árbol (se revisa la cáscara de la fruta y si tiene color café-naranja significa que está madura). Si se recolecta la fruta antes de que alcance la madurez fisiológica, ésta ya no madura, por ser un fruto no climatérico.

\section{Variables analizadas}

Peso fresco de las frutas: peso individual de cada fruto en gramos (g) con balanza de precisión Ohaus Scout Pro (Ohaus Corporation, Parsippany, NJ, USA).

Longitud y diámetro ecuatorial de las frutas: medidas en $\mathrm{mm}$ con calibrador vernier digital (Truper ${ }^{\circledR}$, México).

Color de la pulpa: esta variable se evaluó con un equipo Minolta ${ }^{\circledR}$ Lab marca ColorTec PCM/PSM Catalogo 59730-7 (Konica Minolta Sensing Americas, Ramsey, NJ, USA), que registra los valores de L
$(100=$ blanco puro, $0=$ negro puro) a y $b$ (coordenadas de cromaticidad; $+a=$ rojo, $-a=$ verde; $+b=$ amarillo, $-b=a z u l)$. A partir de los análisis realizados se reporta la luminosidad, ángulo Hue y Croma (Minolta, 1994; Díaz et al., 2001).

Firmeza (Newton): Utilizando el Penetrómetro Digital.

Sólidos solubles totales: utilizando un refractómetro digital marca Atago PAL-1 (Tokio, Japón), 0-53\%.

Acidez titulable: se determinó la acidez por titulación con $\mathrm{NaOH} 0,1 \mathrm{~N}$ y $0,01 \mathrm{~N}$, y tres gotas de fenolftaleína al $1 \%$, en una muestra de $10 \mathrm{~mL}$ de pulpa y agua destilada en una relación 10:50.

Los resultados de la caracterización fueron analizados mediante análisis de varianza de $P \leq 0,05$.

\section{RESULTADOS Y DISCUSIÓN}

Los productores mencionan que existen árboles frutales de reciente plantación hasta frutales de 100 años 
de edad (Tab. 2) como caimito y zapote domingo, que siguen siendo productivos. La edad reportada, la calcularon a partir de información de sus padres y abuelos. Estos resultados indican la tradición generacional de cultivar frutales criollos y la conservación de la biodiversidad de especies de interés y, la renovación permanente de los árboles cuando el frutal no produce.

Se encontraron un total de 326 árboles para Congregación Juana Moza y 272 para el Ejido, siendo más abundante la guaya en ambas comunidades con 114 y 77 árboles y el menos abundante fue el caimito con 63 y 24 árboles para Congregación y Ejido Juana Moza, respectivamente.

\section{Tabla 2. Número total y edad de los árboles de cinco espe- cies frutales criollas en solares de dos comunida- des de Tuxpan, México.}

\begin{tabular}{|l|c|c|c|c|}
\hline \multirow{2}{*}{ Especie } & \multicolumn{2}{|c|}{$\begin{array}{c}\text { Congregación Juana } \\
\text { Moza }\end{array}$} & \multicolumn{2}{c|}{ Ejido Juana Moza } \\
\cline { 2 - 5 } & $\begin{array}{c}\text { Edad } \\
\text { (años) }\end{array}$ & $\begin{array}{c}\text { Total de } \\
\text { árboles }\end{array}$ & $\begin{array}{c}\text { Edad } \\
\text { (años) }\end{array}$ & $\begin{array}{c}\text { Total de } \\
\text { árboles }\end{array}$ \\
\hline Caimito & $4-100$ & 63 & $2-60$ & 24 \\
\hline Chalahuite & $1-53$ & 58 & $2-40$ & 71 \\
\hline Guaya & $2-55$ & 114 & $3-40$ & 77 \\
\hline Jobo & $3-40$ & 35 & $3-28$ & 49 \\
\hline $\begin{array}{l}\text { Zapote } \\
\text { domingo }\end{array}$ & $1-60$ & 56 & $2-100$ & 51 \\
\hline Total & & 326 & & 272 \\
\hline
\end{tabular}

El destino de la producción de los frutales es el autoconsumo reportado por el 83,5 y $83,3 \%$ de los entrevistados en Congregación y Ejido Juana Moza, sin embargo también reportan la venta en el mercado local, el 64,5 y $65,0 \%$ de los entrevistados. Lo que representa que un porcentaje importante vende sus frutales en fresco. Se reportan otros usos específicos por frutal. El jobo se utiliza para hacer bebidas refrescantes con sabor a fruta y preparar curados (su elaboración consiste en la adición de frutos maduros a una bebida alcohólica, la cual puede ser: tequila, ron, aguardiente, mezcal, entre otras, las cuales se dejan reposar de 1-12 meses antes de ser consumidas, permitiendo que adquieran el sabor y aroma de la fruta). El zapote domingo se utiliza para preparar bebidas refrescantes y conservas (mermeladas y jalea). Las semillas del chalahuite se consumen después de un proceso de cocción con sal, lo cual ha sido reportado por Ronquillo (1988) y Ruiz et al. (2004).

Los productores reportan que las especies frutales, además son utilizadas como cercas vivas y de sombrío para otras plantas de ornato y diferentes lugares del hogar.

\section{Variables físico-químicas}

Los frutos de caimito tanto verdes como morados, no presentaron diferencias estadísticas significativas $(P>0,05)$ en las variables peso, longitud, diámetro,

Tabla 3. Peso, longitud y diámetro ecuatorial de frutales criollas de Ejido y Congregación Juana Moza en Tuxpan, México.

\begin{tabular}{|c|l|c|c|c|}
\hline Comunidad & \multicolumn{1}{|c|}{ Fruto } & Peso $(\mathrm{g})$ & Longitud $(\mathrm{mm})$ & Diámetro $(\mathrm{mm})$ \\
\hline \multirow{5}{*}{ Congregación Juana Moza } & Caimito verde & $112,6 \pm 27.27$ & $59,1 \pm 4,9$ & $58,2 \pm 3,8$ \\
\cline { 2 - 5 } & Caimito morado & $128,85 \pm 25,72$ & $58,53 \pm 5,37$ & $61,13 \pm 4,43$ \\
\cline { 2 - 5 } & Guaya & $11,41 \pm 0,82^{*}$ & $27,63 \pm 1,13$ & $25,99 \pm 1,02^{*}$ \\
\cline { 2 - 5 } & Zapote domingo & $570,46 \pm 187,79$ & $95,29 \pm 9,94^{*}$ & $106,01 \pm 13,62$ \\
\cline { 2 - 5 } & Chalahuite & $291,96 \pm 61,36$ & $23,49 \pm 2,99$ & $4,73 \pm 0,79$ \\
\cline { 2 - 5 } & Jobo & $9,99 \pm 3,35$ & $33,16 \pm 3,50$ & $22,69 \pm 2,69$ \\
\hline \multirow{5}{*}{ Ejido Juana Moza } & Caimito verde & $116,6 \pm 29,7$ & $57,0 \pm 5,5$ & $58,6 \pm 5,6$ \\
\cline { 2 - 5 } & Caimito morado & $116,45 \pm 28,94$ & $55,55 \pm 4,93$ & $59,28 \pm 4,51$ \\
\cline { 2 - 5 } & Guaya & $12,86 \pm 1,69^{*}$ & $28,43 \pm 1,46$ & $27,54 \pm 1,63^{*}$ \\
\cline { 2 - 5 } & Zapote domingo & $531,32 \pm 91,38$ & $88,81 \pm 5,06^{*}$ & $105,99 \pm 7,26$ \\
\cline { 2 - 5 } & Chalahuite & $259,18 \pm 75,86$ & $21,22 \pm 5,65$ & $4,91 \pm 0,40$ \\
\cline { 2 - 5 } & Jobo & $10,29 \pm 2,65$ & $33,04 \pm 4,15$ & $22,57 \pm 2,00$ \\
\hline
\end{tabular}

*: significancia $(P \leq 0,05)$. 
firmeza, grados Brix y color (Tab. 3); presentando valores similares para las dos comunidades, con valores medios de peso de 112 y $116 \mathrm{~g}$. Al respecto, Hernández et al. (2009) reportaron valores de 185,5 y 200,2 g para las comunidades Ejido Juana Moza y Zapotal Zaragoza, respectivamente, por lo que puede deducirse que los tamaños medios de los frutos varían de un año a otro, encontrados frutos de menor tamaño, lo cual puede atribuirse a condiciones climáticas cambiantes (variaciones en precipitación y temperatura).

Los frutos de guaya presentaron diferencias estadísticas $(P \leq 0,05)$ en peso y diámetro ecuatorial, presentando valores numéricos mayores en Ejido Juana Moza (Tab. 3). Pérez et al. (2008), reportan que la ubicación de los frutos de Melicoccus bujigatus en el árbol, la temperatura y el tiempo de almacenamiento, así como la interacción ubicación-temperatura producen efectos significativos $(P \leq 0,05)$ en el peso, diámetro polar y ecuatorial del fruto.

Los resultados para zapote domingo muestran que los frutos son similares en ambas comunidades en las variables peso, y diámetro ecuatorial, con valores medios de 531-570 g (valores máximos de 622,00-758,25 g) y 105-106 cm entre Ejido y Congregación Juana Moza, respectivamente, aunque se encontraron diferencias significativas en longitud de 88,8-95,3 cm entre Ejido y Congregación Juana Moza, respectivamente (Tab. 3). Los valores medios de peso y diámetro encontrados difieren con los valores medios reportados por Cedeño et al. (2010), quienes reportan peso promedio de fruto de zapote domingo de $653,23 \mathrm{~g}$ y $18,60 \mathrm{~cm}$ de diámetro, pero dentro del rango encontrado en los frutos evaluados.

En chalahuite el peso de fruto presentó valores medios de 292 y 259 g y una longitud/diámetro de $23 / 5$ y 21/5 cm para Congregación y Ejido Juana Moza, respectivamente (Tab. 3). Estos resultados concuerdan con los obtenidos por Alfaro et al. (2010), con valores promedios de 332,5; 340,25 y 261,9 g en un estudio realizado para evaluar el estado nutrimental de la harina de semillas de chalahuite, en tres comunidades de Guatemala. Otro uso potencial posible de esta especie para la zona de estudio.

Para la variable acidez de caimito verde y morado se encontraron diferencias estadísticas significativas (Tab. 4), presentando valores mayores el caimito morado 1,39-1,52\% entre Congregación y Ejido Juana
Moza, respectivamente. Al respecto Zambrano et al. (2013), obtuvieron valores promedio de 0,31\% de acidez en caimito verde y $0,19 \%$ en morado y $6,28{ }^{\circ} \mathrm{Brix}$ en los dos tipos de caimito, mientras que en el presente estudio se encontraron valores de ${ }^{\circ}$ Brix de 12-13 ${ }^{\circ}$ Brix para caimito verde y 14,07-15,25 para caimito morado en las dos comunidades estudiadas (Tab. 3). Aunque es importante mencionar que el estudio de Zambrano et al. (2013) se desarrolló en una huerta de la Universidad de los Andes en Venezuela.

Para la variable acidez titulable, firmeza y color de los frutos de guaya se presentan valores similares en las dos comunidades (Tab. 4); el color de los frutos fue intenso y con luminosidad alta, en las dos comunidades, 51,61; 45,9 y 44,66 para Congregación Juana Moza y 54,00; 50,02 y -79,19 para el Ejido. Esto debido a que los árboles muestreados se encontraban en su mayoría con luz directa del sol por ser árboles sin competencia (sin obstrucción de luz por árboles más grandes). Pérez et al. (2009) encontraron que los niveles de color fueron menores $(P \leq 0,05)$ en los frutos provenientes de la parte inferior de los árboles, lo cual indica que estos frutos tuvieron menor intensidad de color y menor luminosidad a los cosechados en la parte superior y media del árbol (superior: 26,$70 ;-2,17$ y 5,53 ; media: 26,55 ; $-2,18$ y 5,46 ; inferior: 26,$22 ;-2,05$ y 5,04$)$, por la disposición con respecto a la radiación solar.

En zapote domingo la concentración de grados Brix presentaron diferencias estadísticas con valores medios de 6,47 a 13,49 en las dos comunidades siendo mayores y por ende más dulces los frutos provenientes del Ejido Juana Moza (Tab. 4). Cedeño et al. (2010) reportan que durante el almacenamiento a temperaturas de $-30^{\circ} \mathrm{C}$ los sólidos solubles totales, acidez y color permanecieron estables en los frutos de zapote domingo.

En las variables de firmeza, sólidos solubles totales o grados Brix, acidez y color de chalahuite se encontraron valores muy similares para las dos comunidades en estudio (Tab. 4). En jobo, el análisis de varianza muestra diferencias estadísticas significativas $(P \leq 0,05)$ para la variables firmeza, grados Brix y acidez. Los sólidos solubles totales y acidez fue mayor en el Ejido Juana Moza, pero se presentó mayor firmeza en Congregación Juana Moza (Tab. 4). 
Tabla 4. Firmeza, grados Brix, acidez y color en frutales criollos de Ejido y Congregación Juana Moza en Tuxpan, México.

\begin{tabular}{|c|c|c|c|c|c|c|c|}
\hline \multirow{2}{*}{ Comunidad } & \multirow{2}{*}{ Frutal } & \multirow{2}{*}{ Firmeza (N) } & \multirow{2}{*}{ Grados Brix } & \multirow{2}{*}{ Acidez (\%) } & \multicolumn{3}{|c|}{ Color } \\
\hline & & & & & $\mathrm{L}$ & C & $\mathrm{H}$ \\
\hline \multirow{6}{*}{$\begin{array}{l}\text { Congregación } \\
\text { Juana Moza }\end{array}$} & Caimito verde & $3,31 \pm 2,28$ & $12,03 \pm 4,86$ & ${ }^{*} 1,35 \pm 0,27$ & 52,10 & 30,40 & $-78,74$ \\
\hline & Caimito morado & $3,88 \pm 2,11$ & $15,25 \pm 3,82$ & $1,39 \pm 0,33$ & 36,36 & 15,52 & 32,58 \\
\hline & Guaya & $2,34 \pm 0,87$ & $18,13 \pm 3,21^{*}$ & $6,59 \pm 0,58$ & 51,61 & 45,98 & $44,66^{*}$ \\
\hline & Zapote domingo & $10,77 \pm 2,65$ & $6,47 \pm 4,95^{*}$ & $3,96 \pm 1,22$ & 51,40 & 24,88 & 69,89 \\
\hline & Chalahuite & $11,4 \pm 2,6$ & $16,0 \pm 2,0$ & $0,8 \pm 0,1$ & 51,4 & 32,2 & 31,77 \\
\hline & Jobo & $0,74 \pm 0,53^{*}$ & $14,91 \pm 0,97^{*}$ & ${ }^{*} 18,23 \pm 1,53$ & 47,37 & 15,08 & $39,36^{*}$ \\
\hline \multirow{6}{*}{$\begin{array}{l}\text { Ejido Juana } \\
\text { Moza }\end{array}$} & Caimito verde & $3,89 \pm 2,87$ & $13,45 \pm 2,30$ & ${ }^{*} 1,14 \pm 0,25$ & 50,54 & 30,00 & $-47,14$ \\
\hline & Caimito morado & $3,59 \pm 1,71$ & $14,07 \pm 1,07$ & $1,52 \pm 0,28$ & 34,51 & 12,87 & 33,28 \\
\hline & Guaya & $2,94 \pm 1,88$ & $15,91 \pm 1,86^{*}$ & $6,68 \pm 0,79$ & 54,00 & 50,02 & $-79,19^{*}$ \\
\hline & Zapote domingo & $11,64 \pm 3,48$ & $13,49 \pm 0,92^{*}$ & $3,81 \pm 1,14$ & 50,04 & 26,55 & 67,72 \\
\hline & Chalahuite & $11,7 \pm 2,5$ & $16,2 \pm 1,22$ & $0,8 \pm 0,1$ & 50,0 & 35,0 & $-32,0$ \\
\hline & Jobo & $1,55 \pm 0,60^{*}$ & $14,08 \pm 0,72^{*}$ & ${ }^{*} 10,36 \pm 0,97$ & 46,83 & 14,41 & $35,15^{*}$ \\
\hline
\end{tabular}

$*$ : significancia $(P \leq 0,05)$.

\section{CONCLUSIONES}

El aprovechamiento que los productores le dan a caimito verde y morado (Chrysophyllum cainito L.), chalahuite (Inga paterno Harms), guaya (Melicoccus bijugatus Jacq.), zapote domingo (Mammea americana L.) y jobo (Spondias mombin L.) manejados a nivel de huerto o solar es la obtención de frutos para complementar la dieta familiar y para la obtención de recursos económicos mediante la venta de la fruta fresca, en el mercado local o mediante la elaboración de bebidas refrescantes, conservas, "curados" y otros subproductos, durante la temporada de producción que va de abril a septiembre.

Las especies frutales estudiadas tienen características de madurez, época de fructificación, peso, diámetro, color y usos similares, lo cual puede ser útil para la planeación de un manejo y aprovechamiento regional sustentable de las especies estudiadas.

Las variables grados Brix y acidez presentan diferencias significativas, pese a que las dos comunidades estudiadas se encuentran muy cercanas entre sí y comparten características edafoclimáticas similares, pero difieren en cuanto a ubicación de los solares con respecto a la fuentes de agua. Los solares de Congregación Juana Moza se localizan a los costados del río Tuxpan, lo que les confiere humedad de manera permanente y un mayor contenido de agua.
El aprovechamiento de las especies frutales criollas representa una tradición transgeneracional, es un patrimonio heredado, apropiado y recreado, de evidente importancia ecológica, económica y social en las escalas familiares y local, de valor regional, mediante la transmisión de conocimiento referente al uso y conservación de la diversidad de frutales, mediante su conservación, implícita en una amplia diversidad de edades de las poblaciones nativas, con aprovechamiento y renovación continua de las especies útiles para el consumo humano, venta e incluso intercambio, valorizando el uso de éstas especies como sombra, cercos, leña, entre otros.

Las cinco especies de frutales criollos estudiadas son aprovechadas y comercializadas en fresco o bien como conservas, mermeladas, dulces o curados (jobo y zapote domingo), sin embargo éstas modalidades han sido limitadas a la región y a la época de producción y no se reporta su disponibilidad para cubrir la demanda durante todo el año, o para abastecer un mercado mayor.

Los resultados encontrados en la encuesta y las variables evaluadas para contribuir a la caracterización físico-química pueden ser útiles para el productor y para promover el cultivo de manera extensiva de los frutales estudiados, buscando con ello abastecer el mercado local de manera permanente o bien su expansión a un mercado mayor que permita la generación de opciones para mejorar la económica del productor, 
así como para su confrontación respecto a estudios de preferencia de la demanda del mercado local.

Conflicto de intereses: el manuscrito fue preparado y revisado con la participación de los autores, quienes declaran no tener algún conflicto de interés que coloquen en riesgo la validez de los resultados aquí presentados.

\section{REFERENCIAS BIBLIOGRÁFICAS}

Alfaro, M.A., P. Figueroa, C. Arriaga y R. Bressani. 2010. Valor nutricional de la harina de semillas de Inga. Estudios preliminares para su incorporación a la dieta de la población rural. Rev. Univ. Val. Guatem. 19, 45-57.

Álvarez, V.J.E., I. Alia, V. López, C.M. Acosta, M. Andrade, L.M.T. Colinas, E.I. Delgado y T.O. Villegas. 2006. Caracterización de frutos de caimito (Chrysophyllum cainito L.), en el estado de Morelos. Rev. Chapingo Ser. Hortic. 12(2), 217-221.

Ambrosio, M. y S. Avendaño. 1999. Catálogo de plantas útiles del municipio de Misantla, Veracruz. México. La Ciencia y el Hombre 31, 43-87.

Borys, M.W. y B.H. Leszczyñska. 2001. El potencial frutícola de la República Mexicana. Fundación Salvador Sánchez Colín; Cictamex, Toluca, México.

Cedeño, E.M., K.V. Viteri y A.M. Costa. 2010. Estudio del comportamiento de la pulpa congelada y del aceite de semillas obtenido de dos variedades diferentes de mamey Colocarpum mammosum (mamey colorado) y Mammea americana (mamey cartagena). Trabajo de grado. Facultad de Ingeniería en Mecánica y Ciencias de Producción, Escuela Superior Politécnica del Litoral, Guayaquil, Ecuador.

Hernández, M.L., D. Hernández, P. Elorza, M. López y M.A. López. 2009. Caracterización de frutos de caimito (Chrysophyllum cainito L.) en el estado de Veracruz, México. Rev. Cient. UDO Agríc. 9(1), 70-73.
Lascurain, M., S. Avendaño y A. Niembro. 2010. Guía de frutos silvestres comestibles en Veracruz. Fondo Sectorial para la Investigación, el Desarrollo y la Innovación Tecnológica Forestal, Conafort-Conacyt, México.

López, E.J., R. Szeszko, J. Rescala, y A. Beltrán. 2012. The flora of the Tenancingo-Malinalco-Zumpahuacán protected natural area, State of Mexico, Mexico. Harv. Pap. Bot. 17(1), 65-167. Doi: 10.3100/025.017.0113

National Research Council (NRC). 1989. Lost crops of the Incas. Little known plants of the Andes with promise for worldwide cultivation. National Academy Press, Washington, D.C., USA.

Peña, L.E.G. y V. Ruiz. 2003. Vitaminas hidrosolubles en fitorecursos alimenticios de comunidades étnicas de tabasco. Universidad y Ciencia 38(19), 85-91.

Pérez, H.C., M. Gómez y J. Vila. 2008. Evaluación de los parámetros de calidad en frutos de mamoncillo ( $M e$ licoccus bijuga L.). Características químicas. Iberoam. Tecnol. Postcos. 9(1), 7-15.

Pérez, H.C., M. Gómez y J. Vila. 2009. Características físicas de frutos de mamón (Melicoccus bijugatus Jacq.) según su ubicación en el árbol y el almacenamiento. Bioagro 21(3), 189-194.

Rodríguez, G., F. Leal y B. Naranjo. 2012. Situación actual de los cultivos frutales de mayor importancia en Venezuela. Rev. Fac. Agron. Ed. Esp., 207-221.

Ruiz, V., G. Peña, C. Lau, F. Maldonado, M. Ascencio y M.A. Guadarrama. 2004. Macronutrimentos de fitorecursos alimenticios de especies aprovechadas por grupos étnicos en Tabasco, México. Universidad y Ciencia No. Esp. 1, 27-31.

Vernooy, R. 2003. Semillas generosas. Mejoramiento participativo de plantas. IDRC-CRDI, Ottawa, Canadá.

Zambrano, J., N. Montilla, R. Riveros, I. Quintero, M. Maffei, A. Valera y W. Materano. 2013. Caracterización de frutos de caimito (Chrysophyllum cainito L.) variedades verde y morado e influencia de la época de cosecha en la calidad de los frutos. Revista Academia (Venezuela) 12(27), 287-294. 